International Journal of Bifurcation and Chaos, Vol. 8, No. 10 (1998) 2031-2040

(C) World Scientific Publishing Company

\title{
SYNCHRONIZATION OF HYPERCHAOTIC CIRCUITS VIA CONTINUOUS FEEDBACK CONTROL WITH APPLICATION TO SECURE COMMUNICATIONS
}

\author{
MICHELE BRUCOLI*, DONATO CAFAGNA and LEONARDA CARNIMEO \\ Dipartimento di Elettrotecnica ed Elettronica, \\ Politecnico di Bari, Via E. Orabona, 4 - 70125 Bari, Italy \\ GIUSEPPE GRASSI \\ Dipartimento di Matematica, Università di Lecce 73100 Lecce, Italy
}

Received August 8, 1997; Revised March 11, 1998

\begin{abstract}
In this paper a synthesis technique for the synchronization of hyperchaotic circuits with application to secure communications is developed. It is well known that information-bearing signals can be considered as causes of perturbations, when a secure communication system based on a masking technique is implemented. Starting from this consideration, in this paper a feedback control scheme is developed to guarantee synchronization between transmitter and receiver. The control scheme involves as many state variables in the feedback as the number of information signals to be transmitted. After deriving the nonlinear dynamics of the synchronization error system, the feedback matrix is determined by finding a suitable Lyapunov function and by imposing the conditions which assure the global asymptotic stability of the origin of the error system. The method is illustrated by considering hyperchaotic circuits constituted by coupled Chua's oscillators, for which analytic expressions of the feedback gains are derived.
\end{abstract}

\section{Introduction}

In recent years several methods devoted to the synchronization of chaotic circuits for secure communications have been investigated [Ogorzalek, 1993; Kocarev et al., 1992; Halle et al., 1993; Cuomo et al., 1993; Lozi \& Chua, 1993; Wu \& Chua, 1993, 1994; Milanovic \& Zaghloul, 1996]. In particular, by considering that chaotic signals are broadband noiselike waveforms, very difficult to predict, these techniques are based on a chaotic masking of information-bearing signals. The information signal is successively recovered at the receiver which has been previously synchronized with the transmitter.

The problem of synchronizing chaotic circuits for secure communication systems is not simple due to an important aspect which has to be taken into account. Chaotic systems are very sensitive to perturbations, which could cause the loss of synchronization when a masking technique is implemented. Taking into account that informationbearing signals can certainly be considered as causes of perturbations, some attractive synchronization methods have been developed, which keep the transmitter equivalent to the receiver by introducing a feedback mechanism involving the information signal itself [Wu \& Chua, 1993, 1994; Milanovic \& Zaghloul, 1996]. This interesting idea has been extended in this paper to the synchronization of hyperchaotic circuits in secure communication systems. The choice of circuits characterized by a much more complex dynamics, such as hyperchaotic ones, is motivated by the

*E-mail: brucoli@poliba.it 
availability of several chaotic signals with a consequent improvement of the security of the communication system [Brucoli et al., 1996, 1997].

At first, a synchronization scheme for secure communications constituted of $n$-dimensional transmitting and receiving systems which exhibit hyperchaotic behavior, is illustrated. The transmitter is driven by the information signals, whereas the receiver is driven both by the state variables and the information signals. More in detail, the receiver is controlled by continuous feedback signals given by the difference between the transmitted signals and the state variables of the receiver. The transmitter is kept equivalent to the receiver by feeding the information-bearing signals back to the transmitter. This linear feedback action is characterized by a feedback gain matrix.

Successively, simple considerations lead individualization of the dynamics of the synchronization error system. The feedback matrix is determined in the proposed design method by finding a suitable Lyapunov function and imposing the conditions which assure the global asymptotic stability of the origin of the error system [Wu \& Chua, 1994; Slotine \& Li, 1991; Schweizer et al., 1995; Suykens et al., 1997]. In this way, a perfect synchronization is achieved between the transmitter and the receiver. The suggested technique is applied to a communication scheme where both transmitter and receiver are constituted of a pair of bidirectionally coupled Chua's oscillators. The use of coupled Chua's oscillators is due to the fact that this system is a well-known prototype for chaos analysis [Madan, 1993] and it has already been implemented as an integrated circuit device [Cruz \& Chua, 1993]. For this system, analytic expressions are derived for the elements of the feedback gain matrix.

Finally, simulation results are carried out to show the validity of the proposed synchronization technique.

\section{Synchronization via Feedback Control with Application to Secure Communications}

A design method is illustrated to achieve synchronization between all the state variables of two hyperchaotic circuits. This approach is exploited to synthesize a secure communications system. To this purpose, an $n$-dimensional transmitting system exhibiting hyperchaotic behavior is considered, which can be formally described by the following equation [Wu \& Chua, 1994]:

$$
\dot{x}=\varphi(x, x, s)
$$

where $x=\left[x_{1}, x_{2}, \ldots, x_{n}\right]^{T} \in \mathcal{R}^{n}$ is the state vector of the transmitter, $s=\left[s_{1}, s_{2}, \ldots, s_{n}\right]^{T} \in \mathcal{R}^{n}$ is the vector of the information-bearing waveforms, and $\varphi: \mathcal{R}^{n} x \mathcal{R}^{n} x \mathcal{R}^{n} \rightarrow \mathcal{R}^{n}$ is a vector function.

Now, the following equation has to be considered for the receiver:

$$
\dot{x}^{\prime}=\eta\left(x^{\prime}, x, s\right)
$$

where $x^{\prime}=\left[x_{1}^{\prime}, x_{2}^{\prime}, \ldots, x_{n}^{\prime}\right]^{T} \in \mathcal{R}^{n}$ is the state vector of the receiver, whereas $\eta: \mathcal{R}^{n} x \mathcal{R}^{n} x \mathcal{R}^{n} \rightarrow \mathcal{R}^{n}$ is a vector function. The vectors $x$ and $s$ can be considered as driving signals for system (2).

In order to exploit feedback control techniques [Hu et al., 1995] for assuring synchronization between transmitter and receiver, it is assumed that Eqs. (1) and (2) can be rewritten as:

$$
\begin{aligned}
\dot{x} & =h(x)+M s \\
\dot{x}^{\prime} & =h\left(x^{\prime}\right)+M\left[(x+s)-x^{\prime}\right]
\end{aligned}
$$

where $h: \mathcal{R}^{n} \rightarrow \mathcal{R}^{n}$ is a vector function and the matrix $M$ is chosen as an (nxn)-diagonal feedback gain matrix with the aim of simplifying the structure of the controller to be designed. Namely, the adoption of a full gain matrix $M$ would lead to a great number of feedback loops, which is a serious drawback from the viewpoint of the controller realization and reliability [Suykens et al., 1997]. Note that in Eq. (3b) a continuous feedback action has been adopted for the receiver by considering the difference between the transmitted signals and the state variables of the receiving system. Moreover, in order to achieve perfect synchronization between the transmitter and the receiver, the transmitter is kept equivalent to the receiver by feeding the vector $M s$ back to the transmitter. A block diagram illustrating the proposed approach is reported in Fig. 1.

It is worth noting that transmitting the vector $x+s$ instead of a function of the system state might be less secure. In fact, interesting cryptographic methods for chaotic circuits have recently been proposed which aim at preventing an intruder from synchronizing with the transmitter [Yang et al., 1997]. However, since in this paper the attention is focused on the synchronization method for hyperchaotic systems rather than on the security of the communication scheme, a simpler masking technique has been considered. 


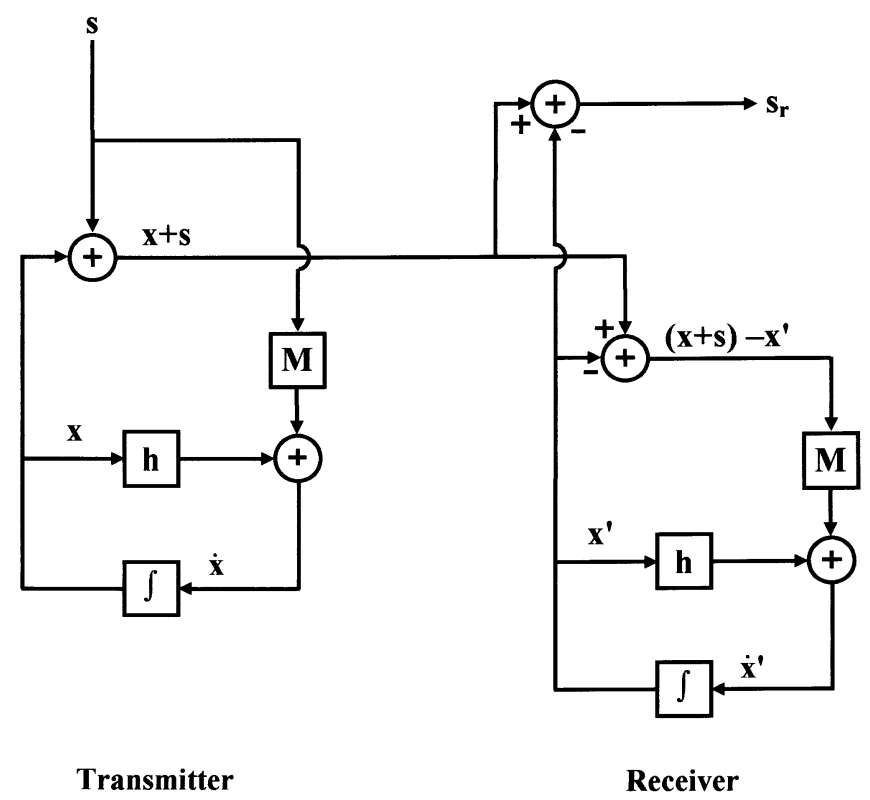

Fig. 1. Block diagram illustrating the proposed feedback control scheme for synchronization.

Now, by defining the vector of the synchronization errors as [Schweizer et al., 1995]:

$$
e(t)=x(t)-x^{\prime}(t)
$$

and, taking into account Eqs. (3a) and (3b), the following equation describing the error dynamics is obtained:

$$
\dot{e}(t)=h(x)-h\left(x^{\prime}\right)-M e
$$

where $e=\left[e_{1}, \ldots, e_{i}, \ldots, e_{n}\right]^{T}$ is the error vector, being $e_{i}=x_{i}-x_{i}^{\prime}$, for $i=1, \ldots, n$. The main idea of the proposed design method consists in assuring the global asymptotic stability of the origin of the error dynamics by means of a suitable Lyapunov function $V(e)$. Namely, the unknown elements $M_{i j}$ of the feedback matrix $M$ can be computed by imposing that:

(i) $V(e)$ be positive definite

(ii) $\dot{V}(e)$ be negative definite

(iii) $V(e) \rightarrow \infty$ for $\|e\| \rightarrow \infty$ (that is, $V(e)$ be radially unbounded)

If the above conditions are satisfied, then $e->$ 0 as $t->\infty$ and, therefore, $x->x^{\prime}$ as $t->\infty$. In this way, synchronization is achieved. As a consequence, the information signals can be recovered at the receiver as:

$$
s_{r}(t)=\left[(x(t)+s(t))-x^{\prime}(t)\right] \approx s(t)
$$

It should be noted that there are many physical systems that can be properly described by a mathe- matical model in which the linear part and the nonlinear one appear separated [Wu \& Chua, 1993]. For this reason, the suggested method is here applied to that class of transmitting and receiving systems whose state equations can be expressed as:

$$
\begin{aligned}
\dot{x} & =A x+g(x)+M s \\
\dot{x}^{\prime} & =A x^{\prime}+g\left(x^{\prime}\right)+M\left[(x+s)-x^{\prime}\right]
\end{aligned}
$$

where the matrix $A \in \mathcal{R}^{n x n}$ individualizes the linear part of these systems, whereas the function $g$ takes into account all the nonlinearities. The previously shown block diagram (Fig. 1) modifies as indicated in Fig. 2. In this case, the following system of equations for the error dynamics can be obtained:

$$
\dot{e}=A e+g(x)-g\left(x^{\prime}\right)-M e
$$

Now, in order to satisfy the constraint (i), the following candidate Lyapunov function is chosen:

$$
V(e)=\sum_{i=1}^{n} \lambda_{i} e_{i}{ }^{2}
$$

where $\lambda_{i}>0, i=1, \ldots, n$. Note that the function (8) represents a diagonal Lyapunov function, which may lead to conservative results. Although this choice may imply a slow convergence of the error to the origin [Khalil, 1992], nevertheless it gives the opportunity of deriving a simple control scheme. 


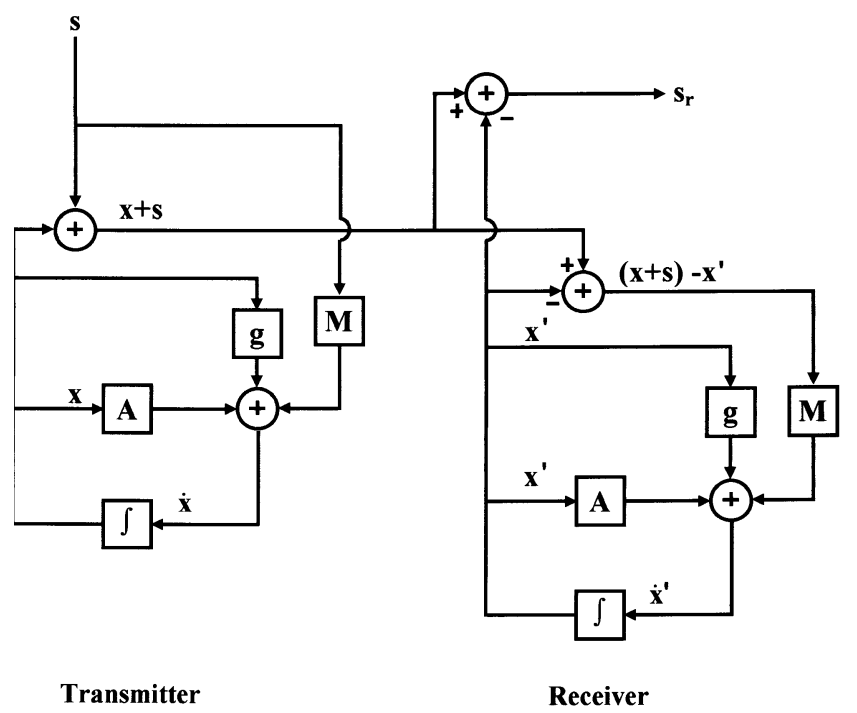

Fig. 2. Block diagram illustrating the suggested secure communication system related to Eq. (6). form:

Namely, by expressing $\dot{V}(e)$ as a quadratic

$$
\dot{V}(e)=-e^{T} \psi e
$$

where $\psi \in \mathcal{R}^{n x n}$, the unknown feedback parameters $M_{i j}$ can be determined by imposing constraints (ii) and (iii) to $\dot{V}(e)$ and $V(e)$, respectively.

\section{Design of Synchronized Coupled Chua's Oscillators for Secure Communications}

Following the considerations of the preceding section a communication scheme is considered where the transmitter and the receiver equations are well represented by the state equations in the form (6a) and (6b), respectively. To this purpose, a transmitter constituted by a pair of bidirectionally coupled Chua's oscillators is here considered. As is well known, this circuit is characterized by a rich variety of dynamical behaviors [Anishchenko et al., 1994; Kapitaniak et al., 1994]. Namely, depending on the choice of circuit parameters, this system can exhibit different types of hyperchaotic attractors as well as chaos-hyperchaos intermittency. By assuming that two signals $s_{1}$ and $s_{4}$ have to be transmitted by means of two different channels and that, accordingly, two chaotic state variables have to be used as masking signals, the transmitter state equation is:

$$
\dot{x}=A x+g(x)+M s
$$

where

$$
\begin{aligned}
& x=\left[x_{1}, x_{2}, x_{3}, x_{4}, x_{5}, x_{6}\right]^{T} \\
& g(x)=\left[-\alpha f\left(x_{1}\right), 0,0,-\alpha_{0} f_{0}\left(x_{4}\right), 0,0\right]^{T} \\
& s=\left[s_{1}, 0,0, s_{4}, 0,0\right]^{T} \\
A= & {\left[\begin{array}{cccccc}
-\alpha & \alpha & 0 & 0 & 0 & 0 \\
1 & (-1-K) & 1 & 0 & K & 0 \\
0 & -\beta & -\gamma & 0 & 0 & 0 \\
0 & 0 & 0 & -\alpha_{0} & \alpha_{0} & 0 \\
0 & H & 0 & 1 & (-1-H) & 1 \\
0 & 0 & 0 & 0 & -\beta_{0} & -\gamma_{0}
\end{array}\right] }
\end{aligned}
$$

with

$$
\begin{gathered}
f\left(x_{1}\right)=b x_{1}+(a-b)\left(\left|x_{1}+1\right|-\left|x_{1}-1\right|\right) / 2 \\
f_{0}\left(x_{4}\right)=b_{0} x_{4}+\left(a_{0}-b_{0}\right)\left(\left|x_{4}+1\right|-\left|x_{4}-1\right|\right) / 2
\end{gathered}
$$

For the considered configuration, $\alpha, \beta, \alpha_{0}, \beta_{0}, K$ and $H$ are positive constants, whereas $a$ and $a_{0}, b$ and $b_{0}$ are real constants. The parameters $K$ and $H$ individualize the bidirectional coupling between the two Chua's circuits [Anishchenko et al., 1994].

Since two signals have to be transmitted using two different channels, the feedback gain matrix $M$ in Eq. (5) can be properly chosen as:

$$
M=\operatorname{diag}\left[M_{11}, 0,0, M_{44}, 0,0\right]
$$


Then, the state equation of the receiver $i=1, \ldots, 6$, and becomes:

$$
\dot{x}^{\prime}=A x^{\prime}+g\left(x^{\prime}\right)+M\left[(x+s)-x^{\prime}\right]
$$

where

$$
\begin{aligned}
x^{\prime} & =\left[x_{1}^{\prime}, x_{2}^{\prime}, x_{3}^{\prime}, x_{4}^{\prime}, x_{5}^{\prime}, x_{6}^{\prime}\right]^{T} \\
g\left(x^{\prime}\right) & =\left[-\alpha f\left(x_{1}^{\prime}\right), 0,0,-\alpha_{0} f_{0}\left(x_{4}^{\prime}\right), 0,0\right]^{T}
\end{aligned}
$$

with

$$
f\left(x_{1}^{\prime}\right)=b x_{1}^{\prime}+(a-b)\left(\left|x_{1}^{\prime}+1\right|-\left|x_{1}^{\prime}-1\right|\right) / 2
$$

$$
f_{0}\left(x_{4}^{\prime}\right)=b_{0} x_{4}^{\prime}+\left(a_{0}-b_{0}\right)\left(\left|x_{4}^{\prime}+1\right|-\left|x_{4}^{\prime}-1\right|\right) / 2
$$

Now, the following equation for the error system is obtained:

$$
\dot{e}=A e+d\left(x, x^{\prime}\right)-M e
$$

$$
\begin{aligned}
d\left(x, x^{\prime}\right)= & g(x)-g\left(x^{\prime}\right) \\
= & \left\{-\alpha\left[f\left(x_{1}\right)-f\left(x_{1}^{\prime}\right)\right], 0,0,\right. \\
& \left.-\alpha_{0}\left[f_{0}\left(x_{4}\right)-f_{0}\left(x_{4}^{\prime}\right)\right], 0,0\right\}^{T} .
\end{aligned}
$$

In order to determine the elements $M_{11}$ and $M_{44}$ of the feedback gain matrix $M$, let

$$
\begin{aligned}
V(e)= & \left(\lambda_{1} e_{1}{ }^{2}+\lambda_{2} e_{2}{ }^{2}+\lambda_{3} e_{3}{ }^{2}\right. \\
& \left.+\lambda_{4} e_{4}{ }^{2}+\lambda_{5} e_{5}{ }^{2}+\lambda_{6} e_{6}{ }^{2}\right) / 2
\end{aligned}
$$

be the candidate Lyapunov function with $\lambda_{i}>0$, $i=1, \ldots, 6$.

Since for Eqs. (11) and (13) it results:

$$
\begin{aligned}
& -\left[f\left(x_{1}\right)-f\left(x_{1}^{\prime}\right)\right] /\left(x_{1}-x_{1}^{\prime}\right) \leq \max \{|a|,|b|\} \quad \\
& -\left[f_{0}\left(x_{4}\right)-f_{0}\left(x_{4}^{\prime}\right)\right] /\left(x_{4}-x_{4}^{\prime}\right) \leq \max \left\{\left|a_{0}\right|,\left|b_{0}\right|\right\}
\end{aligned}
$$

where $e=\left[e_{1}, e_{2}, e_{3}, e_{4}, e_{5}, e_{6}\right]^{T}$, being $e_{i}=x_{i}-x_{i}^{\prime}$,

the derivative of $V(e)$ along the system trajectory can be expressed as:

$$
\begin{aligned}
\dot{V}(e) \leq & {\left[\lambda_{1}\left(-\alpha-M_{11}+\alpha \max \{|a|,|b|\}\right) e_{1}{ }^{2}+\left(\alpha \lambda_{1}+\lambda_{2}\right) e_{1} e_{2}-\lambda_{2} e_{2}{ }^{2}\right] } \\
& +\left(\lambda_{2}-\beta \lambda_{3}\right) e_{2} e_{3}-\gamma \lambda_{3} e_{3}{ }^{2}+\left[\lambda_{4}\left(-\alpha_{0}-M_{44}+\alpha_{0} \max \left\{\left|a_{0}\right|,\left|b_{0}\right|\right\}\right) e_{4}{ }^{2}\right. \\
& \left.+\left(\alpha_{0} \lambda_{4}+\lambda_{5}\right) e_{4} e_{5}-\lambda_{5} e_{5}{ }^{2}\right]+\left(\lambda_{5}-\beta_{0} \lambda_{6}\right) e_{5} e_{6}-\gamma_{0} \lambda_{6} e_{6}{ }^{2} \\
& -\left[\lambda_{2} K e_{2}{ }^{2}-\left(K \lambda_{2}-H \lambda_{5}\right) e_{2} e_{5}+\lambda_{5} H e_{5}{ }^{2}\right]
\end{aligned}
$$

Referring to (17), by considering the worst case, it is possible to write $\dot{V}(e)$ as a quadratic form [Suykens et al., 1997]:

$$
\dot{V}(e)=-e^{T} \psi e
$$

where $\psi \in \mathcal{R}^{6 \times 6}$ is a symmetric matrix given by:

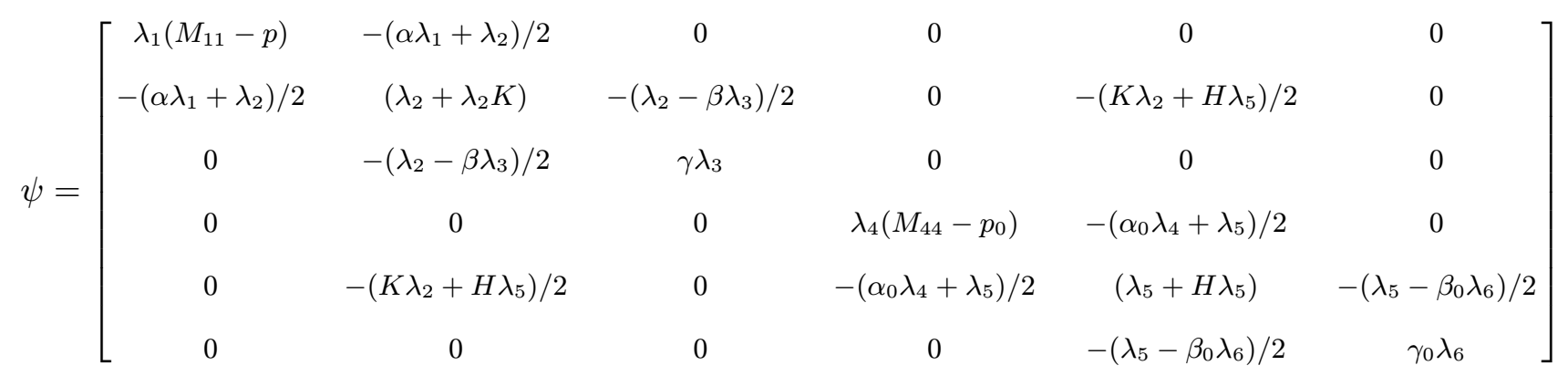


with $p=-\alpha+\alpha \max \{|a|,|b|\}$ and $p_{0}=-\alpha_{0}+$ $\alpha_{0} \max \left\{\left|a_{0}\right|,\left|b_{0}\right|\right\}$. For the considered configuration it can be easily shown that the parameters $p$ and $p_{0}$ are positive. Without loss of generality, it can be assumed that:

$$
\lambda_{3}=\lambda_{2} / \beta \quad \lambda_{6}=\lambda_{5} / \beta_{0}
$$

As a consequence, Eq. (18) becomes:

$$
\dot{V}(e)=-e_{r}^{T} \psi^{1} e_{r}-\gamma \lambda_{2} e_{3}^{2} / \beta-\gamma_{0} \lambda_{5} e_{6}^{2} / \beta_{0}
$$

where $e_{r}=\left[e_{1}, e_{2}, e_{4}, e_{5}\right]^{T}$ is a reduced error vector and $\psi^{1} \in \mathcal{R}^{4 \times 4}$ is the symmetric submatrix obtained from $\psi$ by deleting the third and the sixth rows and the third and the sixth columns, respectively.

By imposing that the matrix $\psi^{1}$ be positive definite, i.e. that all principal minors be strictly positive [Slotine \& Li, 1991], the parameters $\lambda_{i}, i=$ $1, \ldots, 6, M_{11}$ and $M_{44}$ can be derived. To this purpose, by indicating with $\psi_{r}^{1}$ the generic principal minor of $\psi^{1}$, it must be imposed that $\psi_{r}^{1}>0, r=$ $1, \ldots, 4$. In particular, the synthesis procedure is carried out in four steps, where each step is based on the results of the previous one.

For $r=1$, it results

$$
\psi_{1}^{1}=\lambda_{1}\left(M_{11}-p\right)>0
$$

which gives

$$
M_{11}>p
$$

As a consequence, $M_{11}$ has to be chosen as follows:

$$
M_{11}=\alpha(\max \{|a|,|b|\}-1)+\varepsilon_{1}
$$

with $\varepsilon_{1}>0$ to be determined by using the minor $\psi_{2}^{1}$ in the second step.
For $r=2$

$$
\psi_{2}^{1}=\lambda_{1} \lambda_{2}(K+1) \varepsilon_{1}-\left(\alpha \lambda_{1}+\lambda_{2}\right)^{2} / 4>0
$$

From Eq. (23), it results that

$$
\varepsilon_{1}=\left[\left(\alpha \lambda_{1}+\lambda_{2}\right)^{2} / 4 \lambda_{1} \lambda_{2}(K+1)\right]+\delta_{1}
$$

with $\delta_{1}>0$ to be determined.

A necessary condition for the driving system to show a chaotic behavior also in the presence of the feedback action involving the informationbearing signal, consists in choosing values of $\varepsilon_{1}$ which minimize $M_{11}$. This consideration is in accordance with the one reported in $[\mathrm{Wu} \&$ Chua, 1993]. After mathematical manipulations, the values of $\left(\lambda_{1}, \lambda_{2}\right) \in \mathcal{R}^{+} x \mathcal{R}^{+}-\{0,0\}$ which minimize the function

$$
g\left(\lambda_{1}, \lambda_{2}\right)=\left(\alpha \lambda_{1}+\lambda_{2}\right)^{2} / 4 \lambda_{1} \lambda_{2}(K+1)
$$

are found to be [Apostol, 1969]

$$
\lambda_{2}=\alpha \lambda_{1} \quad \forall \lambda_{1}>0
$$

Consequently,

$$
\varepsilon_{1}=\delta_{1}+\alpha /(K+1)
$$

For $r=3$, it results

$$
\psi_{3}^{1}=\lambda_{4}\left(M_{44}-p_{0}\right)\left[\alpha \delta_{1} \lambda_{1}^{2}(K+1)\right]>0
$$

which holds for $M_{44}>p_{0}$ since $\lambda_{1}, \lambda_{4}, \alpha, K, \delta$ are all positive.

As a consequence, the value of the gain $M_{44}$ is:

$$
M_{44}=\alpha_{0}\left(\max \left\{\left|a_{0}\right|,\left|b_{0}\right|\right\}-1\right)+\varepsilon_{4}
$$

with $\varepsilon_{4}>0$ to be determined in the following step. For $r=4$

$$
\begin{aligned}
\psi_{4}^{1}= & {\left[\alpha \delta_{1} \lambda_{1}^{2}(K+1)\right]\left[\lambda_{4} \lambda_{5} \varepsilon_{4}(H+1)-\left(\alpha_{0} \lambda_{4}+\lambda_{5}\right)^{2} / 4\right] } \\
& -\left\{\lambda_{1} \lambda_{4} \varepsilon_{4}\left[\delta_{1}+\alpha /(K+1)\right]\left(K \lambda_{2}+H \lambda_{5}\right)^{2} / 4\right\}>0
\end{aligned}
$$

Taking into account the procedure followed to obtain Eqs. (26) and (27), it can be shown that

$$
\begin{aligned}
& \lambda_{5}=\alpha_{0} \lambda_{4} \quad \forall \lambda_{4}>0 \\
& \varepsilon_{4}=\delta_{4}+\alpha_{0} /(K+1)
\end{aligned}
$$

with $\delta_{4}>0$. Since the previous results hold for every $\lambda_{1}$ and $\lambda_{4}$, these coefficients can be chosen as:

$$
\lambda_{1}=\lambda_{4}=\lambda>0
$$


Thus, inequality (29) becomes:

$$
\psi_{4}^{1}=\left\{\left[\delta_{1} \alpha(K+1)\right]\left[\delta_{4} \alpha_{0}(H+1)\right]-\left[\delta_{1}+\alpha_{0} /(K+1)\right]\left[\delta_{4}+\alpha /(H+1)\right]\left[K \alpha+H \alpha_{0}\right]^{2} / 4\right\} \lambda^{4}>0
$$

The solution of Eq. (33) is given by:

$$
\begin{gathered}
\delta_{1}>\alpha\left(K \alpha+H \alpha_{0}\right) /\left\{(K+1)\left[2 \alpha(K+1)-\left(K \alpha+H \alpha_{0}\right)\right]\right\} \\
\delta_{4}>\alpha_{0}\left(K \alpha+H \alpha_{0}\right) /\left\{(H+1)\left[2 \alpha_{0}(H+1)-\left(K \alpha+H \alpha_{0}\right]\right\}\right.
\end{gathered}
$$

From (34) it can be obtained:

$$
\begin{aligned}
& \varepsilon_{1}=[\alpha /(K+1)]+\alpha\left(K \alpha+H \alpha_{0}\right) /\left\{(K+1)\left[2 \alpha(K+1)-\left(K \alpha+H \alpha_{0}\right)\right]\right\}+\xi_{1} \\
& \varepsilon_{4}=\left[\alpha_{0} /(H+1)\right]+\alpha_{0}\left[K \alpha+H \alpha_{0}\right) /\left\{(H+1)\left[2 \alpha_{0}(H+1)-\left(K \alpha+H \alpha_{0}\right)\right]\right\}+\xi_{4}
\end{aligned}
$$

where $\xi_{1}$ and $\xi_{4}$ are small arbitrary positive constants.

Finally, the results of the proposed synthesis procedure can be summarized as follows:

$$
\begin{aligned}
\forall \lambda_{1}= & \lambda_{4}=\lambda>0 \\
\lambda_{2}= & \alpha \lambda ; \quad \lambda_{5}=\alpha_{0} \lambda ; \quad \lambda_{3}=\alpha \lambda / \beta ; \quad \lambda_{6}=\alpha_{0} \lambda / \beta_{0} \\
M_{11}= & \alpha(\max \{|a|,|b|\}-1)+[\alpha /(K+1)]+\alpha\left(K \alpha+H \alpha_{0}\right) /\{(K+1)[2 \alpha(K+1) \\
& \left.\left.-\left(K \alpha+H \alpha_{0}\right)\right]\right\}+\xi_{1} \\
M_{44}= & \alpha_{0}\left(\max \left\{\left|a_{0}\right|,\left|b_{0}\right|\right\}-1\right)+\left[\alpha_{0} /(H+1)\right]+\alpha_{0}\left(K \alpha+H \alpha_{0}\right) /\left\{( H + 1 ) \left[2 \alpha_{0}(H+1)\right.\right. \\
& \left.\left.-\left(K \alpha+H \alpha_{0}\right)\right]\right\}+\xi_{4}
\end{aligned}
$$

Remark 1. It should be noted that the determined feedback gains $M_{11}$ and $M_{44}$ guarantee the global asymptotic stability of the origin for the error system regardless the waveforms of the information signals, which is an important feature for the secure communication system.

Remark 2. It can be shown [Hale, 1969] that the error vector $e$ converges to zero at least exponentially.

\section{Numerical Example}

In this example both the transmitter and the receiver are constituted by a pair of bidirectionally coupled Chua's oscillators characterized by the following parameter values:

$$
\begin{array}{lcc}
\alpha=10.00, & \beta=14.870, & \gamma=0.03850, \\
a=-1.270, & b=-0.680, & \alpha_{0}=11.00, \\
\beta_{0}=16.357, & \gamma_{0}=0.04235, & a_{0}=-1.397, \\
b_{0}=-0.748, & K=0.25 ; & H=0.275 ; \\
K_{0}=0.25 ; & H_{0}=0.275, &
\end{array}
$$

The initial conditions for the transmitter and the receiver are, respectively:

$$
\begin{aligned}
& x_{1}(0)=0.01 ; \quad x_{4}(0)=0.011 ; \\
& x_{2}(0)=x_{3}(0)=x_{5}(0)=x_{6}(0)=0 \\
& x_{1}^{\prime}(0)=0.011 ; \quad x_{4}^{\prime}(0)=0.012 ; \\
& x_{2}^{\prime}(0)=x_{3}^{\prime}(0)=x_{5}^{\prime}(0)=x_{6}^{\prime}(0)=0
\end{aligned}
$$

The above values of parameters and initial conditions assure the existence of hyperchaotic behavior both to the transmitter and the receiver. The corresponding attractor behavior has been depicted in Fig. 3.

In this example the sinusoidal waveforms $s_{1}(t)=A_{1} \sin \left(2 \pi f_{1} t\right)$ and $s_{4}(t)=A_{4} \sin \left(2 \pi f_{4} t\right)$ with $A_{1}=5 \times 10^{-6}, A_{4}=1 \times 10^{-6}, f_{1}=20 \mathrm{~Hz}$ and $f_{4}=30 \mathrm{~Hz}$ have been considered as informationbearing signals.

By applying the proposed synthesis procedure, the following values of the coefficients of the considered Lyapunov function have been derived: $\lambda_{1}=$ $1 ; \lambda_{2}=10 ; \lambda_{3}=0.6725 ; \lambda_{4}=1 ; \lambda_{5}=11 ; \lambda_{6}=$ 0.6725 . The values of the feedback gains have been determined as: $M_{11}=12.970, M_{44}=15.120$. It 


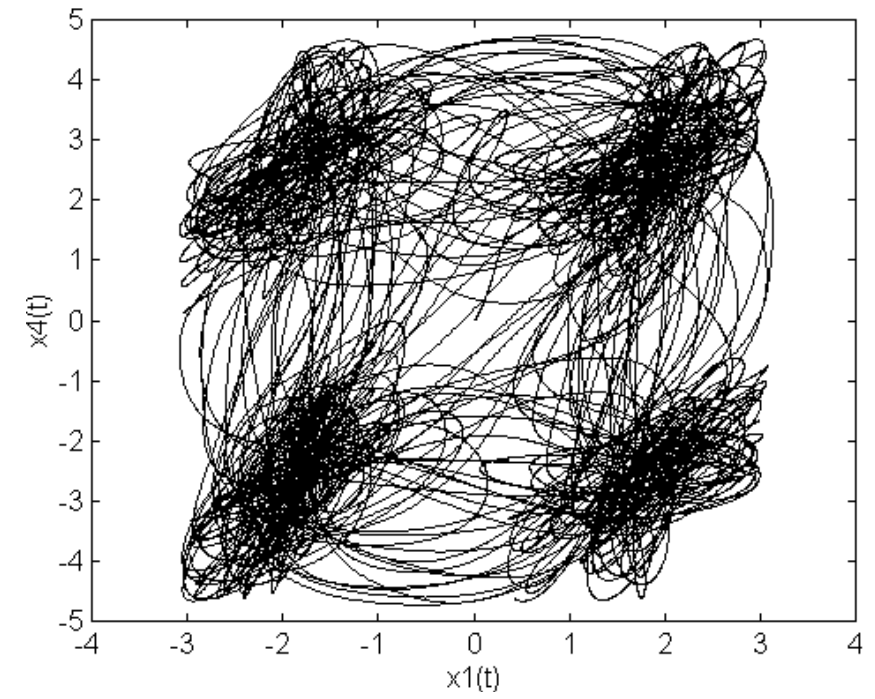

Fig. 3. The double-double scroll attractor.
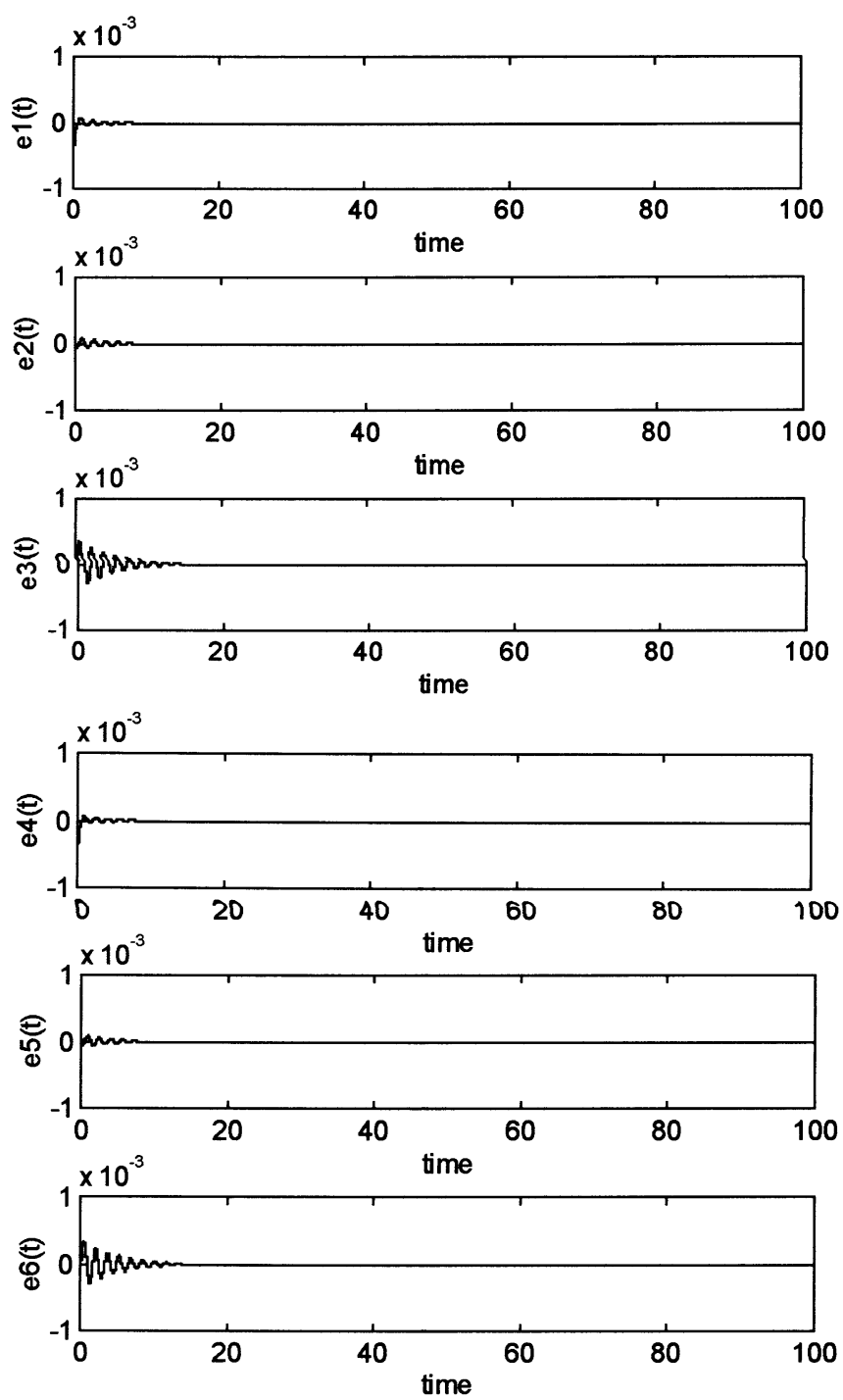

Fig. 4. Time behavior of the synchronization errors $e_{i}(t), i=1, \ldots, 6$, in the presence of feedback control. should be noted that the values of $M_{11}$ and $M_{44}$ have been obtained by considering $\xi_{1}=1 \times 10^{-3}$ and $\xi_{4}=9 \times 10^{-3}$, respectively. Figure 4 shows the time waveforms of the synchronization errors $e_{i}(t), i=$ $1, \ldots, 6$ in the presence of feedback control. The capability of the suggested approach is shown in Figure 5 where, as an example, the trajectory of $e_{1}(t)$ with and without the feedback control has been reported. Figure 5 clearly indicates that synchronization is lost in the absence of feedback control. Figure 6 shows the time waveforms
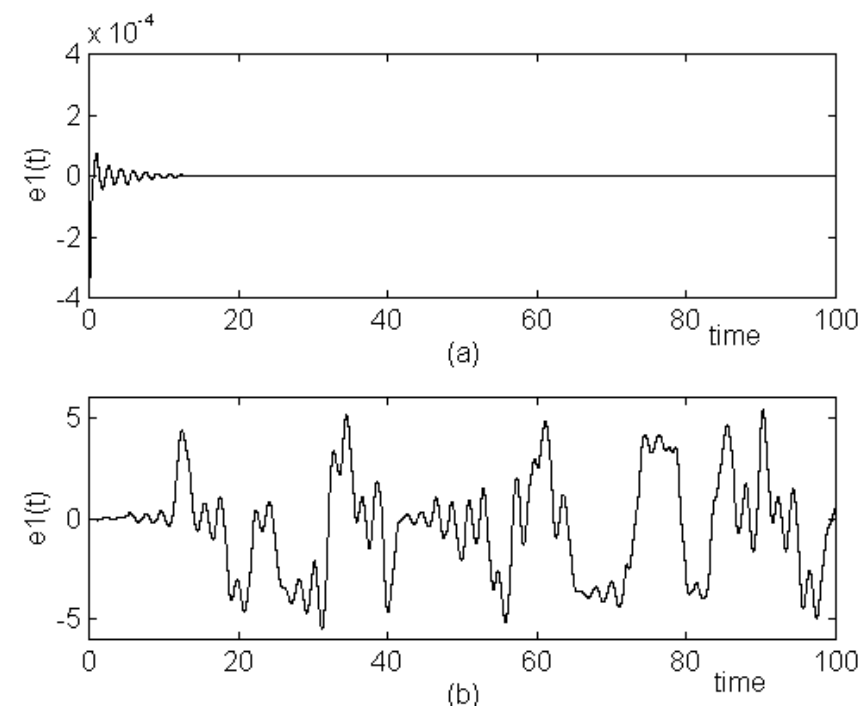

Fig. 5. Comparison between the trajectories of $e_{1}(t)$ with (a) and without (b) feedback control.
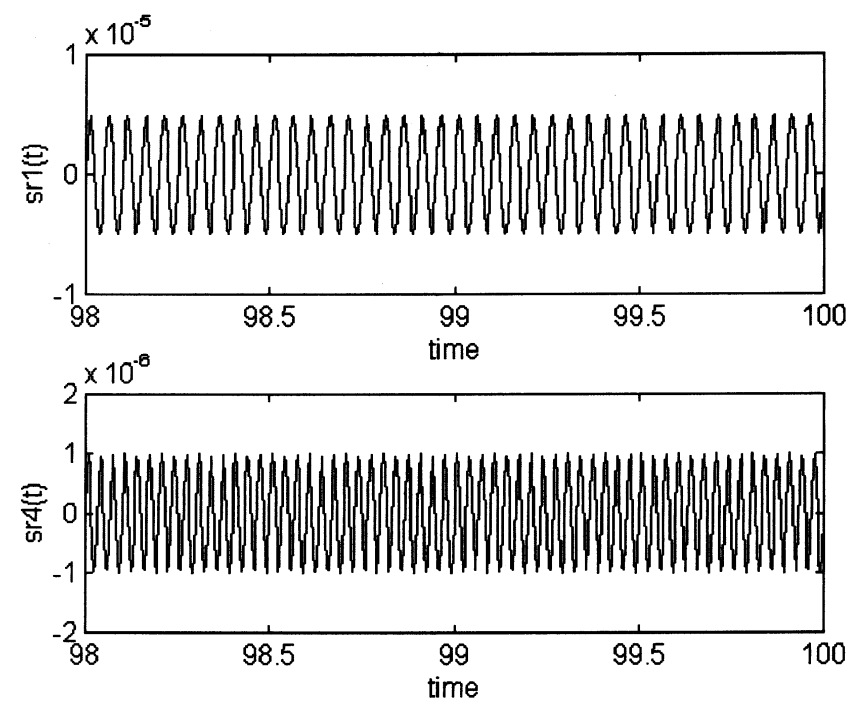

Fig. 6. Time waveforms of the recovered informationbearing signals $s_{r 1}(t), s_{r 4}(t)$. 


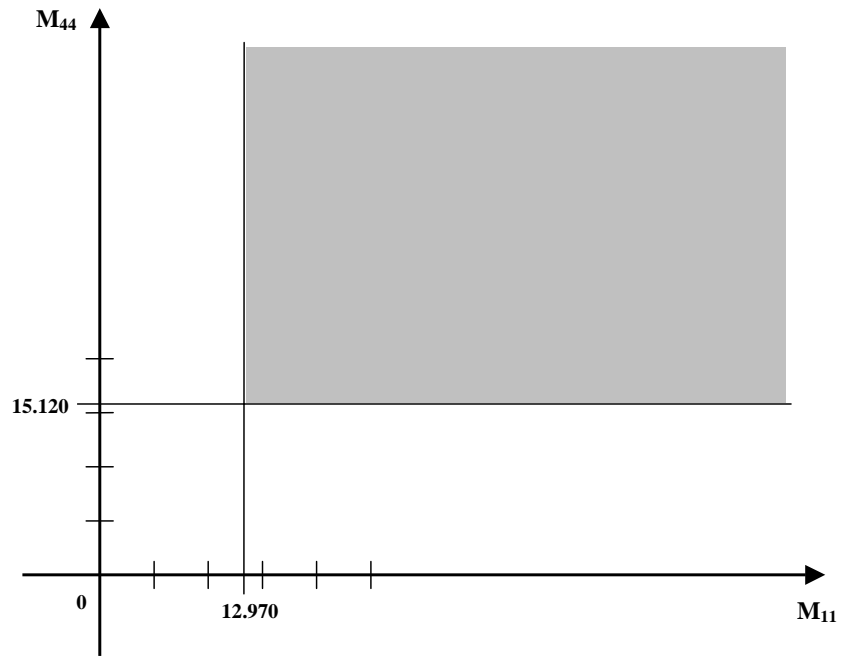

Fig. 7. Synchronization region in the feedback parameter plane.

of the recovered information signals $s_{r 1}(t)$ and $s_{r 4}(t)$. This figure constitutes a further confirm of the capabilities of the proposed method.

Finally, for the given system, Figure 7 shows the region of feedback parameters $M_{11}, M_{44}$ for which synchronization is guaranteed. These results have to be considered as strictly theoretic, since obvious upper limits hold for $M_{11}$ and $M_{44}$ from an implementation viewpoint. The theoretic results have been confirmed by performing many simulations for different pairs $\left(M_{11}, M_{44}\right)$ within the shaded region.

\section{Conclusions}

In this paper a method for synchronizing hyperchaotic circuits via a continuous feedback control has been developed. The suggested approach has been successfully applied to a secure communication scheme where both the transmitter and the receiver are constituted by a pair of coupled Chua's oscillators. Simulation results have been reported to illustrate the capability of the proposed technique.

\section{References}

Anishchenko, V. S., Kapitaniak, T., Safonova, M. A. \& Sosnovzeva, O. V. [1994] "Birth of double-double scroll attractor in coupled Chua circuits," Phys. Lett. A192(3), 207-214.

Apostol, T. M. [1969] Calculus (John Wiley, NY).

Brucoli, M., Cafagna, D., Carnimeo, L. \& Grassi, G.
[1996] "Hyperchaotic circuits for secure communications: An efficient synchronization technique," Proc. 3rd IEEE Int. Conf. Electronic, Circuits and Systems (ICECS'96), Rodos, Greece 2, 639-642.

Brucoli, M., Cafagna, D., Carnimeo, L. \& Grassi, G. [1997] "An efficient technique for signal masking using synchronized hyperchaotic circuits," Int. Workshop on Nonlinear Dynamics of Electronic Systems (NDES'97), Moscow, Russia, pp. 229-234.

Cruz, J. M. \& Chua, L. O. [1993] "An IC chip of Chua's circuit," IEEE Trans. CAS Part II 40(10), 614-625.

Cuomo, K. M., Oppenheim, V. \& Strogatz, S. H. [1993] "Synchronization of Lorenz-based chaotic circuits with applications to communications," IEEE Trans. Circuits Syst. Part II 40(10), 626-633.

Hale, J. K. [1969] Ordinary Differential Equations (Krieger, Huntington, NY).

Halle, K. S., Wu, C. W., Itoh, M. \& Chua, L. O. [1993] "Spread spectrum communication through modulation of chaos," Int. J. Bifurcation and Chaos 3(2), 469-477.

Hasler, M. [1994] "Synchronization principles and applications, tutorials," Proc. IEEE ISCAS '94, pp. 314-326.

Hu, G., Qu, Z. \& He, K. [1995] "Feedback control of chaos in spatiotemporal systems," Int. J. Bifurcation and Chaos 5(4), 901-936.

Kapitaniak, T. \& Chua, L. O. [1994] "Hyperchaotic attractors of unidirectionally-coupled Chua's circuits," Int. J. Bifurcation and Chaos 4(2), 477-482.

Khalil, H. K. [1992] Nonlinear Systems (MacMillan, NY). Kocarev, L., Halle, K. S., Eckert, K., Chua, L. O. \& Parlitz, U. [1992] "Experimental demonstration of secure communications via chaotic synchronization," Int. J. Bifurcation and Chaos 2(3), 709-713.

Lozi, R. \& Chua, L. O. [1993] "Secure communications via chaotic synchronization II: Noise reduction by cascading two identical receivers," Int. J. Bifurcation and Chaos 3(5), 1319-1325.

Madan, R. N. [1993] "Special issue on Chua's circuit: A paradigm for chaos," Int. J. Circuits Syst. Comput. 3(2), 411-430.

Milanovic,V. \& Zaghloul, M. E. [1996] "Improved masking algorithm for chaotic communication systems," Electron. Lett. 32(1), 11-12.

Ogorzalek, M. J. [1993] "Taming chaos-Part I: Synchronization," IEEE Trans. Circuits Syst. Part I 40(10), 693-699.

Schweizer, J., Kennedy, M. P., Hasler, M. \& Dedieu, H. [1995] "Synchronization theorem for a chaotic system," Int. J. Bifurcation and Chaos 5(1), 297-302.

Slotine, J. J. \& Li, W. [1991] Applied Nonlinear Control (Prentice-Hall International, NJ).

Suykens, J. A. K., Curran, P. F. \& Chua, L. O. [1997] 
"Master-slave synchronization using dynamic output feedback," Int. J. Bifurcation and Chaos 7(3), 671-679.

Wu, C. W. \& Chua, L. O. [1993] "A simple way to synchronize chaotic systems with application to secure communication systems," Int. J. Bifurcation and Chaos 3(6), 1619-1627.
Wu, C. W. \& Chua, L. O. [1994] "A unified framework for synchronization and control of dynamical systems," Int. J. Bifurcation and Chaos 4(4), 979-998.

Yang, T., Wu, C. W. \& Chua, L. O. [1997] "Cryptography based on chaotic systems," IEEE Trans. CAS Part I 44(5), 469-472. 